Теорія Ймовір. та Матем. Статист. Вип. 75, 2006
Theor. Probability and Math. Statist.

No. 75, 2007, Pages 139-145 S 0094-9000(08)00720-5

Article electronically published on January 24, 2008

\title{
MIXED EMPIRICAL POINT RANDOM PROCESSES IN COMPACT METRIC SPACES. II
}

UDC 519.21

\author{
YU. I. PETUNIN AND M. G. SEMEǏKO
}

\begin{abstract}
Models of finite simple mixed empirical ordered marked point processes in compact metric spaces are studied in the paper. The processes are constructed from simple samples drawn without replacement from a population. The notion of an ordered marked point process with independent and 1-dependent marks is introduced. Examples of ordered marked point processes with independent and 1dependent marks are given.
\end{abstract}

\section{INTRODUCTION}

A model of a finite simple mixed empirical marked point process is studied in Section 4 . We assume that the space of positions $X$ is equipped with a probability measure $P_{x}$, while the space of marks $K=[a, b] \subset R^{1}$ is equipped with a probability measure $P_{k}$. An arbitrary trajectory of an ordered marked point process is treated as a simple random sample drawn without replacement from a population $Y=X \times K$ and according to the probability measure $P_{\bar{y}}=P_{x} \otimes P_{k}$. We introduce the notion of ordered marked point processes with independent and 1-dependent marks in Section 5. Examples of ordered marked point processes with independent and 1-dependent marks are given in Section 6 ,

\section{MiXED EMPIRICAL ORDERED MARKED POINT PROCESSES IN COMPACT METRIC SPACES}

We recall the definition of a trajectory introduced in Section 1 11 According to this definition, a trajectory $E^{*}$ of a finite simple ordered marked point process $\left(\mathcal{E}^{*}, \mathfrak{X}^{*}, P^{*}\right)$ in a bounded space

$$
\left(Y=X \times K, \mathfrak{A}_{Y}=\mathfrak{A}_{X} \otimes \mathfrak{A}_{K}, \mathfrak{B}_{Y}=\mathfrak{B}_{X} \odot \mathfrak{B}_{K}\right)
$$

is a thinned set of the Cartesian product $Y=X \times K$. If $X$ is a compact metric space and the space of marks $K$ coincides with an interval $[a, b] \subset R^{1}$, then the trajectory $E^{*}$ consists of a finite number of points, namely

$$
E^{*}=\left(\left[x_{1} ; k_{1}\right], \ldots,\left[x_{i} ; k_{i}\right], \ldots,\left[x_{n} ; k_{n}\right]\right) .
$$

The phase space $Y=X \times K$ can be considered to be a compact metric space if the distance between points $\left[x_{1} ; k_{1}\right]$ and $\left[x_{2} ; k_{2}\right]$ is defined by

$$
\rho\left(\left[x_{1} ; k_{1}\right],\left[x_{2} ; k_{2}\right]\right)=\rho_{X}\left(x_{1}, x_{2}\right)+\left|k_{1}-k_{2}\right| .
$$

2000 Mathematics Subject Classification. Primary 60G55.

${ }^{1}$ Editorial Note: This paper is a continuation of [7, with successive numbering of sections and formulas. 
In what follows we assume that $X$ is the population for the random variable $x$ equipped with the probability measure $P_{x}$ defined on the $\sigma$-algebra $\mathfrak{A}_{X}$. The space $K$ is regarded as the population for the random variable $k$ equipped with the probability measure $P_{k}$ defined on the $\sigma$-algebra $\mathfrak{A}_{K}$. Therefore $\left(X, \mathfrak{A}_{X}, P_{x}\right)$ and $\left(K, \mathfrak{A}_{K}, P_{k}\right)$ are the sampling spaces for the random variables $x$ and $k$.

We introduce the product of the probability measures $P_{\bar{y}}=P_{x} \otimes P_{k}$ on the $\sigma$-algebra of Borel sets $\mathfrak{A}_{Y}=\mathfrak{A}_{X} \otimes \mathfrak{A}_{K}$ in the phase space $Y=X \times K$. Then $\left(Y, \mathfrak{A}_{Y}, P_{\bar{y}}\right)$ can be viewed as the sampling probability space of the two-dimensional random variable $\bar{y}=[x ; k]$. This means that the model of a mixed empirical ordered point process studied in Section 3 of [7] can be applied to the ordered marked point process $\left(\mathcal{E}^{*}, \mathfrak{X}^{*}, P^{*}\right)$ in the ordered space $\left(Y=X \times K, \mathfrak{A}_{Y}, \mathfrak{B}_{Y}\right)$ if $X$ is a compact metric space and $K=[a, b]$.

Let $G_{1}$ and $G_{2}$ be two independent stochastic experiments corresponding to the probability spaces $\left(X, \mathfrak{A}_{X}, P_{x}\right)$ and $\left(K, \mathfrak{A}_{K}, P_{k}\right)$, respectively. Then $\bar{G}=\left(G_{1}, G_{2}\right)$ is a "compound" stochastic experiment corresponding to the probability space $\left(Y, \mathfrak{A}_{Y}, P_{\bar{y}}\right)$. A number $n \in Z_{+}$is drawn randomly (according to the probability distribution determined by the generating sequence $\left\{p_{n}^{*}\right\}$ ). Any trajectory

$$
E^{*}=\left(\left[x_{1} ; k_{1}\right], \ldots,\left[x_{i} ; k_{i}\right], \ldots,\left[x_{j} ; k_{j}\right], \ldots,\left[x_{n} ; k_{n}\right]\right)
$$

of a size $n$ of the ordered marked point process is a result of $n$ independent "compound" stochastic experiments $\bar{G}=\left(G_{1}, G_{2}\right)$. Each experiment $\bar{G}$ is a random sampling without replacement of a marked pair $[x ; k]$ from the phase space $Y=X \times K$, where the positions $x_{i}$ belong to the space $X$ (this corresponds to the experiment $G_{1}$ ), while the marks $k_{i}$ belong to the space $K$ (this corresponds to the experiment $G_{2}$ ). Then the trajectory $E^{*}$ can be viewed as a simple sample $\left(x_{i} \neq x_{j}, k_{i} \neq k_{j}, i \neq j\right)$ of a finite size $n$ drawn randomly and without replacement from the population $Y=X \times K$ for the twodimensional random variable $\bar{y}=[x ; k]$ with the joint probability measure $P_{\bar{y}}=P_{x} \otimes P_{k}$. Thus the random elements

$$
\left[x_{1} ; k_{1}\right], \ldots,\left[x_{i} ; k_{i}\right], \ldots,\left[x_{n} ; k_{n}\right]
$$

are independent and identically distributed according to the probability measure $P_{\bar{y}}$. Moreover the mark $k_{i}$ does not depend on the position $x_{i}$ for any marked pair $\left[x_{i} ; k_{i}\right]$, $i=1, \ldots, n$. The projection of the ordered marked point process $\left(\mathcal{E}^{*}, \mathfrak{X}^{*}, P^{*}\right)$ to the space of positions $X$ is a simple mixed empirical ordered point process

$$
(\mathcal{E}, \mathfrak{X}, P)=\operatorname{pr}_{X}\left(\mathcal{E}^{*}, \mathfrak{X}^{*}, P^{*}\right)
$$

in the bounded space of positions $\left(X, \mathfrak{A}_{X}, \mathfrak{B}_{X}\right)$ (see [1]) whose trajectories are simple samples

$$
E=\operatorname{pr}_{X} E^{*}=\left(x_{1}, \ldots, x_{i}, \ldots, x_{j}, \ldots, x_{n}\right), \quad x_{i} \neq x_{j}, i \neq j,
$$

of size $n$ drawn from the population $X$ for the random variable $x$ with probability measure $P_{x}$, while the projection of the ordered marked point process to the space of marks $K$ consists of trajectories $\left(k_{1}, \ldots, k_{i}, \ldots, k_{j}, \ldots, k_{n}\right), k_{i} \neq k_{j}, i \neq j$, that can be viewed as samples of size $n$ drawn randomly and without replacement from the population $K=[a, b]$ for the random variable $k$ with probability measure $P_{k}$.

The process $\left(\mathcal{E}^{*}, \mathfrak{X}^{*}, P^{*}\right)$ is called a strong simple mixed empirical ordered marked point process in the ordered space $\left(Y, \mathfrak{A}_{y}, \mathfrak{B}_{y}\right)$ (see Section 1$)$.

The joint probability distribution $P_{\bar{y}}$ of an arbitrary marked pair, denoted also by $\bar{y}=[x ; k]$ and belonging to the trajectory $E^{*}$ on the metric space $Y=X \times K$, can be expressed in terms of the probability measure $P^{*}$ of the ordered marked point process 
$\left(\mathcal{E}^{*}, \mathfrak{X}^{*}, P^{*}\right)$. Namely,

$$
\begin{aligned}
P_{\bar{y}}\left(B_{Y}\right) & =P_{[x ; k]}\left(B_{X} \times B_{K}\right)=P_{[x ; k]}\left\{[x ; k] \in B_{X} \times B_{K}\right\} \\
& =P^{*}\left\{E^{*}=([x ; k]):[x ; k] \in B_{X} \times B_{K} \mid \mathcal{E}_{1}^{*}\right\} \\
& =P^{*}\left\{E^{*}=([x ; k]): N^{*}\left(E^{*}, B_{X} \times B_{K}\right)=1 \mid \mathcal{E}_{1}^{*}\right\}
\end{aligned}
$$

for all $B_{X} \in \mathfrak{C}_{X}$ and $B_{K} \in \mathfrak{C}_{K}$.

If one considers relation (8) for $B_{K} \equiv K$ and then for $B_{X} \equiv X$, then one obtains the marginal probability distributions $P_{x}\left(B_{X}\right)$ and $P_{k}\left(B_{K}\right)$ of the random variable (position) $x$ on the metric space $X$ and random variable (mark) $k$ on the interval $K=[a, b]$ in terms of the probability measure $P^{*}$. Indeed,

$$
\begin{aligned}
P_{x}\left(B_{X}\right) & =P_{x}\left\{x \in B_{X}\right\}=P_{[x ; k]}\left(B_{X} \times K\right) \\
& =P^{*}\left\{E^{*}=([x ; k]): N^{*}\left(E^{*}, B_{X} \times K\right)=1 \mid \mathcal{E}_{1}^{*}\right\}, \\
P_{k}\left(B_{K}\right) & =P_{k}\left\{k \in B_{K}\right\}=P_{[x ; k]}\left(X \times B_{K}\right) \\
& =P^{*}\left\{E^{*}=([x ; k]): N^{*}\left(E^{*}, X \times B_{K}\right)=1 \mid \mathcal{E}_{1}^{*}\right\}
\end{aligned}
$$

for all $B_{X} \in \mathfrak{C}_{X}$ and $B_{K} \in \mathfrak{C}_{K}$.

This means that $P_{x}\left(B_{X}\right)$ is a probability distribution of the position $x$ if its mark is $k \in K$, while $P_{k}\left(B_{K}\right)$ is the probability distribution of the mark $k$ if its position is $x \in X$.

Since $P_{\bar{y}}$ is the product of the measures $P_{x}$ and $P_{k}$, the probability

$$
\begin{aligned}
l & =P_{\bar{y}}\left(B_{X} \times B_{K}\right)=P_{[x ; k]}\left\{[x ; k] \in B_{X} \times B_{K}\right\}=P_{[x ; k]}\left(B_{X} \times K\right) P_{[x ; k]}\left(X \times B_{K}\right) \\
& =P_{x}\left(B_{X}\right) P_{k}\left(B_{K}\right)=p r
\end{aligned}
$$

is well defined for an arbitrary rectangle $B_{X} \times B_{K} \in \mathfrak{C}_{Y}$, where

$$
p=P_{x}\left(B_{X}\right) \quad \text { and } \quad r=P_{k}\left(B_{K}\right) .
$$

If $n$ is fixed $\left(N^{*}\left(E^{*}, Y\right)=n, n \in Z_{+}\right)$, the conditional distribution of the counting measure $N^{*}\left(E^{*}, B_{X} \times B_{K}\right), B_{X} \times B_{K} \in \mathfrak{C}_{Y}$, of the ordered marked point process $\left(\mathcal{E}^{*}, \mathfrak{X}^{*}, P^{*}\right)$ is the binomial $B(n, l)$ distribution with parameter $l=P_{\bar{y}}\left(B_{X} \times B_{K}\right)$, that is,

$$
P^{*}\left\{E^{*}: N^{*}\left(E^{*}, B_{X} \times B_{K}\right)=k \mid \mathcal{E}_{n}^{*}\right\}=\left(\begin{array}{l}
n \\
k
\end{array}\right) l^{k}(1-l)^{n-k}=\left(\begin{array}{l}
n \\
k
\end{array}\right)(p r)^{k}(1-p r)^{n-k}
$$

where $k=0,1, \ldots, n$.

Using (2), (8), and conditional distribution (9), we evaluate the distribution of the counting measure $N^{*}\left(E^{*}, B_{X} \times B_{K}\right)$ :

$$
P^{*}\left\{E^{*}: N^{*}\left(E^{*}, B_{X} \times B_{K}\right)=k\right\}=\sum_{n=k}^{\infty} p_{n}^{*}\left(\begin{array}{l}
n \\
k
\end{array}\right)(p r)^{k}(1-p r)^{n-k},
$$

where $k \in Z_{+}$and $\left\{p_{n}^{*}\right\}$ is a generating sequence of the distribution $P^{*}$.

\section{Finite Simple ordered MARKED POINT PROCESSES With INDEPENDENT AND 1-DEPENDENT MARKS}

Consider a finite simple ordered marked point process $\left(\mathcal{E}^{*}, \mathfrak{X}^{*}, P^{*}\right)$ in the ordered space

$$
\left(Y=X \times K, \mathfrak{A}_{Y}=\mathfrak{A}_{X} \otimes \mathfrak{A}_{K}, \mathfrak{B}_{Y}=\mathfrak{B}_{X} \odot \mathfrak{B}_{K}\right),
$$

where $X$ is a compact metric space of positions. The space of marks $K=[a, b] \subset R^{1}$ is treated as the population of marks $k$ with an absolutely continuous distribution $P_{k}$. Looking at the trajectory $E^{*}=\left(\left[x_{1} ; k_{1}\right], \ldots,\left[x_{n} ; k_{n}\right]\right)$ of an ordered marked point process, 
one can see a difference between the sequence $E=\left(x_{1}, \ldots, x_{n}\right)$ of positions and that of marks $\left(k_{1}, \ldots, k_{n}\right)$. Namely, if $E$ is a realization of a finite simple ordered point process

$$
(\mathcal{E}, \mathfrak{X}, P)=\operatorname{pr}_{X}\left(\mathcal{E}^{*}, \mathfrak{X}^{*}, P^{*}\right)
$$

in the ordered space of positions $\left(X, \mathfrak{A}_{X}, \mathfrak{B}_{X}\right)$ (see [1]), then $\left(k_{1}, \ldots, k_{n}\right)$ is a simple random sample of a random size $n$ drawn from the population $K$.

Given an arbitrary number $n_{0} \in \mathbb{N}$, vectors $\left(x_{1}, \ldots, x_{n_{0}}\right)=E \subset \mathcal{E}_{n_{0}}$, and sets $B_{K}^{(1)}, \ldots, B_{K}^{\left(n_{0}\right)} \subset \mathfrak{B}_{K}$, where $B_{K}^{(i)} B_{K}^{(j)}=\varnothing, i, j=1, \ldots, n_{0}, i \neq j$, one can evaluate the conditional joint distribution

$$
P_{k_{1} \ldots k_{n_{0}}}\left(B_{K}^{(1)}, \ldots, B_{K}^{\left(n_{0}\right)} \mid \mathcal{E}_{n_{0}}\right)
$$

of marks $k_{1}, \ldots, k_{n_{0}}$ corresponding to given positions $\left(x_{1}, \ldots, x_{n_{0}}\right)=E$, namely

$$
\begin{aligned}
& P_{k_{1} \ldots k_{n_{0}}}\left(B_{K}^{(1)}, \ldots, B_{K}^{\left(n_{0}\right)} \mid \mathcal{E}_{n_{0}}\right)=P_{k_{1} \ldots k_{n_{0}}}\left(B_{K}^{(1)}, \ldots, B_{K}^{\left(n_{0}\right)} \mid\left(x_{1}, \ldots, x_{n_{0}}\right)\right) \\
& =P_{k_{1} \ldots k_{n_{0}}}\left\{k_{1} \in B_{K}^{(1)}, \ldots, k_{n_{0}} \in B_{K}^{\left(n_{0}\right)} \mid\left(x_{1}, \ldots, x_{n_{0}}\right)\right\} \\
& =P^{*}\left\{E^{*}: N^{*}\left(E^{*}, X \times B_{K}^{(1)}\right)=1, \ldots,\right. \\
& \left.N^{*}\left(E^{*}, X \times B_{K}^{\left(n_{0}\right)}\right)=1 \mid\left(x_{1}, \ldots, x_{n_{0}}\right)\right\} \\
& =\mu\left(B_{K}^{(1)}, \ldots, B_{K}^{\left(n_{0}\right)} \mid x_{1}, \ldots, x_{n_{0}}\right),
\end{aligned}
$$

where $\mu\left(B_{K}^{(1)}, \ldots, B_{K}^{\left(n_{0}\right)} \mid x_{1}, \ldots, x_{n_{0}}\right)$ is a probability measure defined on the $\sigma$-algebra $\mathfrak{A}_{K}^{\left(n_{0}\right)}=\mathfrak{A}_{K} \times \cdots \times \mathfrak{A}_{K}\left(n_{0}\right.$ times $)$ and where $x_{1}, \ldots, x_{n_{0}}$ are elements of the $\sigma$-algebra $\mathfrak{A}_{X}$. Putting $B_{K}^{(j)} \equiv K$ in (10) for all $j=1, \ldots, n_{0}, j \neq i$, we get the conditional probability distribution of the mark $k_{i}, i=1, \ldots, n_{0}$ :

$$
\begin{aligned}
& P_{k_{i}}\left(B_{K}^{(i)} \mid \mathcal{E}_{n_{0}}\right)=P_{k_{i}}\left\{k_{i} \in B_{K}^{(i)} \mid\left(x_{1}, \ldots, x_{n_{0}}\right)\right\} \\
& \quad=P_{k_{1} \ldots k_{i-1} k_{i} k_{i+1} \ldots k_{n_{0}}}\left(K, \ldots, K, B_{K}^{(i)}, K, \ldots, K \mid\left(x_{1}, \ldots, x_{n_{0}}\right)\right) \\
& \quad=P^{*}\left\{E^{*}: N^{*}\left(E^{*}, X \times B_{K}^{(i)}\right)=1 \mid\left(x_{1}, \ldots, x_{n_{0}}\right)\right\}=\mu_{i}\left(B_{K}^{(i)} \mid x_{1}, \ldots, x_{n_{0}}\right),
\end{aligned}
$$

where $\mu_{i}\left(B_{K}^{(i)} \mid x_{1}, \ldots, x_{n_{0}}\right)$ is a probability measure defined on the $\sigma$-algebra $\mathfrak{A}_{K}$ and where $x_{1}, \ldots, x_{n_{0}}$ are elements of the $\sigma$-algebra $\mathfrak{A}_{X}$. Given an arbitrary set $B_{K}^{(i)} \in \mathfrak{B}_{K}$, the function $\mu_{i}\left(B_{K}^{(i)} \mid x_{1}, \ldots, x_{n_{0}}\right), x_{1}, \ldots, x_{n_{0}} \in X$, is measurable with respect to the $\sigma$-algebra $\mathfrak{A}_{X}$ (see [5]).

If the neighboring positions

$$
\left(x_{1}, \ldots, x_{i-1}, x_{i+1}, \ldots, x_{n_{0}}\right)
$$

have little influence on the mark $k_{i}, i=1, \ldots, n_{0}$, that is, if the mark $k_{i}$ does not depend on the neighboring positions, then

$$
\begin{aligned}
& P^{*}\left\{E^{*}: N^{*}\left(E^{*}, X \times B_{K}^{(i)}\right)=1 \mid\left(x_{1}, \ldots, x_{n_{0}}\right)\right\} \\
& \quad=P^{*}\left\{E^{*}: N^{*}\left(E^{*}, X \times B_{K}^{(i)}\right)=1 \mid x_{i}\right\}=\mu_{i}\left(B_{K}^{(i)} \mid x_{i}\right),
\end{aligned}
$$

where

$$
\mu_{i}\left(B_{K}^{(i)} \mid x_{i}\right)=P_{k_{i}}\left(B_{K}^{(i)} \mid x_{i}\right)=P_{k_{i}}\left\{k_{i} \in B_{K}^{(i)} \mid x_{i}\right\}
$$

is the probability measure of the transformation of the space $X$ to the space $K$ (see [2]), that is, $\mu_{i}$ is the probability distribution of the mark $k_{i}$ that depends on its position $x_{i}$. 
Also if the influence of positions $\left(x_{1}, \ldots, x_{n_{0}}\right)$ on the mark $k_{i}, i=1, \ldots, n_{0}$, is rather inessential, that is, if the mark $k_{i}$ does not depend on positions $\left(x_{1}, \ldots, x_{n_{0}}\right)$, then

$$
\begin{aligned}
P^{*}\left\{E^{*}: N^{*}\left(E^{*}, X \times B_{K}^{(i)}\right)=1 \mid\left(x_{1}, \ldots, x_{n_{0}}\right)\right\} \\
=P^{*}\left\{E^{*}: N^{*}\left(E^{*}, X \times B_{K}^{(i)}\right)=1\right\}=\mu_{i}\left(B_{K}^{(i)}\right),
\end{aligned}
$$

where

$$
\mu_{i}\left(B_{K}^{(i)}\right)=P_{k_{i}}\left(B_{K}^{(i)}\right)=P_{k_{i}}\left\{k_{i} \in B_{K}^{(i)}\right\}
$$

is a probability distribution of the mark $k_{i}, i=1, \ldots, n_{0}$.

Some applications of marked point processes in stochastic geometry are based on the assumption that the marks are identically distributed random variables (see [4, 6]). Note that this property is involved in the construction of a mixed empirical ordered marked point process (see Section 4). Moreover

$$
\begin{aligned}
\mu_{i}\left(B_{K}^{(i)} \mid x_{1}, \ldots, x_{n_{0}}\right) & =\mu\left(B_{K}^{(i)} \mid x_{1}, \ldots, x_{n_{0}}\right), \quad i=1, \ldots, n_{0}, \\
\mu_{i}\left(B_{K}^{(i)} \mid x_{i}\right) & =\mu\left(B_{K}^{(i)} \mid x_{i}\right), \quad i=1, \ldots, n_{0}, \\
\mu_{i}\left(B_{K}^{(i)}\right) & =\mu\left(B_{K}^{(i)}\right), \quad i=1, \ldots, n_{0} .
\end{aligned}
$$

Definition 14. A finite simple ordered marked point process $\left(\mathcal{E}^{*}, \mathfrak{X}^{*}, P^{*}\right)$ in a bounded space $\left(X \times K, \mathfrak{A}_{X} \otimes \mathfrak{A}_{K}, \mathfrak{B}_{X} \odot \mathfrak{B}_{K}\right)$ is called a process with independent $\mu$-marks if

$$
B_{K}^{(1)}, \ldots, B_{K}^{\left(n_{0}\right)} \subset \mathfrak{B}_{K}
$$

for all $n_{0} \in \mathbb{N}$ where

$$
B_{K}^{(i)} B_{K}^{(j)}=\varnothing, \quad i, j=1, \ldots, n_{0}, i \neq j,
$$

and the marks $k_{1}, \ldots, k_{n_{0}}$ of a random $n_{0}$-dimensional vector $E^{*}=\left(\left[x_{i} ; k_{i}\right]: i=1, \ldots, n_{0}\right)$ are jointly independent random variables in the space $K$, have the probability distribution $\mu\left(B_{K}\right)$, and are such that $k_{1}, \ldots, k_{n_{0}}$ do not depend on their positions $\left(x_{1}, \ldots, x_{n_{0}}\right)$ in the space $X$,

$$
\mu\left(B_{K}^{(1)}, \ldots, B_{K}^{\left(n_{0}\right)} \mid x_{1}, \ldots, x_{n_{0}}\right)=\prod_{i=1}^{n_{0}} \mu\left(B_{K}^{(i)}\right) .
$$

Definition 15. A finite simple ordered marked point process $\left(\mathcal{E}^{*}, \mathfrak{X}^{*}, P^{*}\right)$ in a bounded space $\left(X \times K, \mathfrak{A}_{X} \otimes \mathfrak{A}_{K}, \mathfrak{B}_{X} \odot \mathfrak{B}_{K}\right)$ is called a process with 1 -dependent $\mu$-marks if

$$
B_{K}^{(1)}, \ldots, B_{K}^{\left(n_{0}\right)} \subset \mathfrak{B}_{K}
$$

for all $n_{0} \in \mathbb{N}$ where

$$
B_{K}^{(i)} B_{K}^{(j)}=\varnothing, \quad i, j=1, \ldots, n_{0}, i \neq j,
$$

the marks $k_{1}, \ldots, k_{n_{0}}$ of a random $n_{0}$-dimensional vector $E^{*}=\left(\left[x_{i} ; k_{i}\right]: i=1, \ldots, n_{0}\right)$ are conditionally jointly independent random variables [3]:

$$
\mu\left(B_{K}^{(1)}, \ldots, B_{K}^{\left(n_{0}\right)} \mid x_{1}, \ldots, x_{n_{0}}\right)=\prod_{i=1}^{n_{0}} \mu_{i}\left(B_{K}^{(i)} \mid x_{i}\right)=\prod_{i=1}^{n_{0}} \mu\left(B_{K}^{(i)} \mid x_{i}\right)
$$

such that every mark $k_{i}, i=1, \ldots, n_{0}$, has conditional probability measure $\mu_{i}\left(B_{K}^{(i)} \mid x_{i}\right)$ that depends on a single parameter, namely on its position $x_{i} \in X$. Thus this conditional probability measure belongs to a one-parameter family of probability distributions $\left\{\mu\left(B_{K} \mid x\right): x \in X\right\}:$

$$
\mu_{i}\left(B_{K}^{(i)} \mid x_{i}\right)=\mu\left(B_{K}^{(i)} \mid x_{i}\right)
$$


Definition 16. A finite simple ordered marked point process $\left(\mathcal{E}^{*}, \mathfrak{X}^{*}, P^{*}\right)$ in a bounded space $\left(X \times K, \mathfrak{A}_{X} \otimes \mathfrak{A}_{K}, \mathfrak{B}_{X} \odot \mathfrak{B}_{K}\right)$ is called a process with 1-dependent marks if

$$
B_{K}^{(1)}, \ldots, B_{K}^{\left(n_{0}\right)} \subset B_{K}
$$

for all $n_{0} \in \mathbb{N}$ where

$$
B_{K}^{(i)} B_{K}^{(j)}=\varnothing, \quad i, j=1, \ldots, n_{0}, i \neq j,
$$

the marks $k_{1}, \ldots, k_{n_{0}}$ of a random $n_{0}$-dimensional vector $E^{*}=\left(\left[x_{i} ; k_{i}\right]: i=1, \ldots, n_{0}\right)$ are conditionally jointly independent random variables [3],

$$
\mu\left(B_{K}^{(1)}, \ldots, B_{K}^{\left(n_{0}\right)} \mid x_{1}, \ldots, x_{n_{0}}\right)=\prod_{i=1}^{n_{0}} \mu_{i}\left(B_{K}^{(i)} \mid x_{i}\right)
$$

and the conditional probability distribution $\mu_{i}\left(B_{K}^{(i)} \mid x_{i}\right)$ of any mark $k_{i}, i=1, \ldots, n_{0}$, in the space $K$ depends only on its position $x_{i} \in X$.

\section{EXAMPLES OF ORDERED MARKED POINT PROCESSES WITH INDEPENDENT AND 1-DEPENDENT MARKS}

Example $1([2])$. It is often the case in hydrology that atmospheric precipitates lead to the peak exceedances above the basic level of a river. Let $\left(x_{1}, \ldots, x_{n}\right)$ be the random moments of peak exceedances above the basic level in the interval $X=[O, T] \subset R_{+}^{1}$. One can assume that the points $\left(x_{1}, \ldots, x_{n}\right)$ form a realization $E$ of some ordered point process $(\mathcal{E}, \mathfrak{X}, P)$ in the space of positions $X$ and that $n$ is an integer nonnegative random variable (the number of peak exceedances above the basic level in the interval $X$ ) that does not depend on $\left(x_{1}, \ldots, x_{n}\right)$. The height above the basic flow at the moment $x_{i}$ is denoted by $k_{i}, i=1, \ldots, n$. We also assume that the flow drops below the base level between two successive exceedances. It is clear that $k_{i}$ treated as a random variable (mark) assuming values in the space of marks $K=(0, b]$ depends on the position $x_{i}$ only, that is, $k_{i}=k_{i}\left(x_{i}\right)$, and does not depend on other exceedances $k_{1}, \ldots, k_{i-1}, k_{i+1}, \ldots, k_{n}$. The pairs $\left[x_{i} ; k_{i}\right]$ form a trajectory $E^{*}=\left(\left[x_{1} ; k_{1}\right], \ldots,\left[x_{n} ; k_{n}\right]\right)$ of some ordered marked point process $\left(\mathcal{E}^{*}, \mathfrak{X}^{*}, P^{*}\right)$ in the phase space $X \times K=[O, T] \times(0, b]$. If all marks $k_{i}$ have the same conditional probability measure $\mu_{i}\left(B_{K}^{(i)} \mid x_{i}\right)$ belonging to a one parameter family of probability distributions $\mu_{i}\left(B_{K}^{(i)} \mid x_{i}\right)=\mu\left(B_{K}^{(i)} \mid x_{i}\right), i=1,2, \ldots$, then $\left(\mathcal{E}^{*}, \mathfrak{X}^{*}, P^{*}\right)$ is an ordered marked point process with 1-dependent $\mu$-marks.

Example 2 (Mixed empirical Poisson semispherical segment process). Denote by $\mathcal{A}$ an ordered semispherical segment stochastic process defined on the unit two-dimensional Euclidean sphere $S^{2}$ with trajectories

$$
E_{\mathcal{A}}=\left(Q_{1}\left(u_{1}\left(\varphi_{1}, \theta_{1}\right), a_{1}\right), \ldots, Q_{n}\left(u_{n}\left(\varphi_{n}, \theta_{n}\right), a_{n}\right)\right),
$$

where $u_{i}\left(\varphi_{i}, \theta_{i}\right)$ are the centers of segments, $\left(\varphi_{i}, \theta_{i}\right)$ are spherical coordinates of the centers, $a_{i}$ are their angle diameters, $a_{i} \in K=[O, A]$, and $A \ll \pi, i=1, \ldots, n$ (see [4]). We regard an ordered semispherical segment stochastic process $\mathcal{A}$ as an ordered marked point stochastic process $\left(\mathcal{E}_{\mathcal{A}}^{*}, \mathfrak{X}_{\mathcal{A}}^{*}, P_{\mathcal{A}}^{*}\right)$ with trajectories $E_{\mathcal{A}}^{*}=\left(\left[u_{1} ; a_{1}\right], \ldots,\left[u_{n} ; a_{n}\right]\right)$ in the ordered space $\left(S^{2} \times K, \mathfrak{A}_{S^{2}} \otimes \mathfrak{A}_{K}, \mathfrak{B}_{S^{2}} \odot \mathfrak{B}_{K}\right)$. We assume that every trajectory $E_{\mathcal{A}}^{*}$ is obtained as a result of the following random experiment. Let $G_{1}$ and $G_{2}$ be two independent random experiments corresponding to the probability spaces $\left(S^{2}, \mathfrak{A}_{S^{2}}, P_{u}\right)$ and $\left(K, \mathfrak{A}_{K}, P_{a}\right)$, respectively. Then $\bar{G}=\left(G_{1}, G_{2}\right)$ is a "compound" random experiment corresponding to the probability space $\left(S^{2} \times K, \mathfrak{A}_{S^{2}} \otimes \mathfrak{A}_{K}, P_{u} \otimes P_{a}\right)$ (see Section 4). 
A number $n \in Z_{+}$is drawn randomly according to the probability distribution generated by the Poisson sequence

$$
\left\{p_{n}: p_{n}=\frac{\lambda^{n}}{n !} e^{-\lambda}, \lambda>0\right\}
$$

(see Section 3). Then every trajectory $E_{\mathcal{A}}^{*}$ of the ordered marked point process of size $n$ is obtained as a result of $n$ independent repetitions of the "compound" experiment $\bar{G}$ being the random sampling without replacement of a marked pair $\left[u_{i} ; a_{i}\right]$ from the phase space $S^{2} \times K$ : the positions $u_{i}$ are drawn from the space $S^{2}$ (experiment $G_{1}$ ), while the marks $k_{i}$ are drawn from the space $K$ (experiment $G_{2}$ ). Thus the process $\left(\mathcal{E}_{\mathcal{A}}^{*}, \mathfrak{X}_{\mathcal{A}}^{*}, P_{\mathcal{A}}^{*}\right)$ is a a finite strictly simple mixed empirical Poisson ordered marked point process with independent $P_{a}$-marks.

The stochastic process $\mathcal{A}$ corresponding to the ordered marked point process

$$
\left(\mathcal{E}_{\mathcal{A}}^{*}, \mathfrak{X}_{\mathcal{A}}^{*}, P_{\mathcal{A}}^{*}\right)
$$

is called a mixed empirical Poisson stochastic process of segments.

\section{BIBLIOGRAPHY}

1. Yu. I. Petunin and N. G. Seměko, A random process of segments on a two-dimensional Euclidean sphere. I, Teor. Veroyatnost. i Mat. Statist. 39 (1988), 107-113; English transl. in Theory Probab. Math. Statist. 39 (1989), 129-135. MR947940 (89g:60170)

2. A. F. Karr, Point Processes and their Statistical Inference, 2nd ed., Marcel Dekker, New York, 1991. MR:1113698 (92f:62116)

3. G. Last and A. Brandt, Marked Point Processes on the Real Line: the Dynamic Approach, Springer-Verlag, New York, 1995. MR.1353912 (97c:60126)

4. Yu. I. Petunin and N. G. Semeǐko, Random cap process and generalized Wickell problem on the surface of a sphere, Serdica 17 (1991), 81-91. MR1148300(93c:60010)

5. A. Prekopa, On secondary processes generated by a random point distribution of Poisson type, Ann. Univ. Sci. Budapest. Eötvös. Sect. Math 1 (1958), 153-170. MR0119243 (22:10009)

6. D. Stoyan, W. S. Kendall, and J. Mecke, Stochastic Geometry and its Application, 2nd ed., John Wiley \& Sons, New York, 1987. MR895588 (88j:60034a)

7. Yu. I. Petunin and N. G. Semeǐko, Mixed empirical stochastic point processes in compact metric spaces. I, Teor. Imovirnost. ta Mat. Statist. 74 (2006), 99-109; English transl. in Theory Probab. Math. Statist. 74 (2007), 113-123. MR2321193

Faculty for Cybernetics, National Taras Shevchenko University, Academician Glushkov Avenue, 6, Kyiv 03127, Ukraine

E-mail address: vm214@dcp.kiev.ua

Department of Higher Mathematics, Kyiv National University for Economy, Peremogy Avenue, 54/1, Kyiv, 03057, Ukraine

E-mail address: semejko@ukr.net

Received 13/APR/2005

Translated by N. SEMENOV 